\title{
Urinary miRNA-29a-3p levels are associated with metabolic parameters via regulation of IGF1 in patients with metabolic syndrome
}

\author{
XIHUA LIN ${ }^{1,2}$, CHENG LUO $^{1}$, DONGJUAN HE ${ }^{3}$, ERIK MATRO ${ }^{4}$, \\ QILONG $\mathrm{CHEN}^{4}, \mathrm{HONG} \mathrm{LI}^{1}$ and JIAQIANG ZHOU ${ }^{1}$
}

\begin{abstract}
${ }^{1}$ Department of Endocrinology and ${ }^{2}$ Biomedical Research Center and Key Laboratory of Biotherapy of Zhejiang Province, The Affiliated Sir Run Run Shaw Hospital, College of Medicine, Zhejiang University, Hangzhou, Zhejiang 310016; ${ }^{3}$ Department of Endocrinology, The Third Hospital of Quzhou, Quzhou, Zhejiang $324003 ;{ }^{4}$ College of Medicine, Zhejiang University, Hangzhou, Zhejiang 310058, P.R. China
\end{abstract}

Received September 18, 2018; Accepted February 19, 2019

DOI: $10.3892 /$ br.2019.1195

\begin{abstract}
Circulating microRNAs (miRNAs or miRs) have been demonstrated to serve as diagnostic and prognostic biomarkers in metabolic syndrome (MetS). The role of urinary miRNAs in MetS diagnosis remains unknown. Here, elevated miR-29a-3p levels were observed in urine samples of patients with MetS compared with control subjects using a microarray analysis ( $\mathrm{n}=4 /$ group) and validation via reverse transcription-quantitative polymerase chain reaction ( $n=40$ /group). Associations between urinary miR-29a-3p levels and parameters associated with metabolism, such as adiposity, insulin resistance, lipid profiles and hepatic enzymes were further assessed. Multiple linear regression analyses revealed that urinary miR-29a-3p levels were independently correlated with fasting insulin $(\beta=0.561 ; \mathrm{P}<0.001)$, high density lipoprotein-cholesterol $(\beta=0.242 ; \mathrm{P}<0.001)$ and body mass index $(\beta=-0.141 ; \mathrm{P}<0.05)$. The area under the receiver operating characteristic curve was 0.776 and miR-29a-3p had a diagnostic value for MetS with $68.2 \%$ sensitivity and $77.3 \%$ specificity. Furthermore, insulin-like growth factor 1 was identified as a
\end{abstract}

Correspondence to: Professor Jiaqiang Zhou, Department of Endocrinology, The Affiliated Sir Run Run Shaw Hospital, College of Medicine, Zhejiang University, 3 East Qingchun Road, Hangzhou, Zhejiang 310016, P.R. China

E-mail: zjq8866@zju.edu.cn

Abbreviations: MetS, metabolic syndrome; IGF1, insulin-like growth factor 1; WC, waist circumference; BMI, body mass index; FPG, fasting plasma glucose; FINS, fasting insulin; HOMA-IR, homeostasis model assessment-insulin resistance; SBP, systolic blood pressure; TG, triglyceride; HDL-c, high density lipoprotein-cholesterol; SFA, abdominal subcutaneous fat area; VFA, visceral fat area

Key words: urinary miR-29a-3p, metabolic syndrome, insulin-like growth factor 1 target of miR-29a-3p by searching bioinformatics databases and was validated by dual-luciferase reporter and western blot assays. In conclusion, elevated urinary miR-29a-3p levels were positively associated with MetS and demonstrated to have a potential value as biomarkers in the diagnosis of MetS. The findings provided a better understanding of the role of urinary miRNAs in pathogenesis of MetS.

\section{Introduction}

Metabolic syndrome (MetS) describes a complex metabolic condition, involving abnormal adipose deposition and function, dyslipidemia and hyperglycemia (1). To identify novel therapeutic strategies, microRNAs (miRNAs or miRs) have received attention as potential targets due to their involvement in gene networks regulating the metabolism, including lipid and glucose homeostasis (2). Increasing evidence suggests that circulating miRNAs are involved in the regulation of obesity, diabetes and MetS (3-5). Evaluation of connections between circulating miRNA levels with MetS and insulin sensitivity may lead to novel insight into the pathophysiology of the condition.

Mammalian circulating miRNAs are packaged inside lipid or lipid-protein complexes, such as microvesicles, exosomes or apoptotic bodies (6). Urinary miRNAs are present in cells originating from the kidney or in cells that infiltrated the renal tissue and were excreted into the urine (7). Additionally, miRNAs bound to proteins or incorporated in extracellular vesicles may be released from kidney cells or enter the urine by glomerular filtration (8). Previous literature has demonstrated that endogenous urinary miRNAs are sufficiently stable and quantifiable to serve as clinical biomarkers (9). Mitchell et al (10) reported that the abundance of miRNAs in urine and plasma were strongly correlated.

Considering that clinical specimens of urinary samples are more plentiful in most clinical sample repositories than plasma samples, urinary samples are suitable for miRNA investigations to replace the need for the analysis of certain blood-based biomarkers (11). In diabetic rodent models and 
patients with diabetes, an increase in miR-29 is reported for skeletal muscle, liver, pancreas and white adipose tissues (12-16). Liang et al (17) reported that miR-29a is associated with decreased fasting blood glucose levels through negatively regulating hepatic gluconeogenesis in $\mathrm{db} / \mathrm{db}$ diabetic and diet-induced obese mouse models. Interestingly, an increase in miR-29 levels is observed in the serum of children diagnosed with type 1 and adults diagnosed with type 2 diabetes mellitus (18). In the current study it was assessed if a high level of urinary miR-29a-3p correlated with the incidence of MetS. The present study aimed to investigate the potential prognostic value and regulatory mechanism of urinary miR-29a-3p level in patients with MetS, which may allow clinicians to identify a more appropriate treatment for patients to improve therapeutic outcomes.

\section{Materials and methods}

Study subjects. The study was conducted between March and May 2010 in the Caihe community of Hangzhou (Zhejiang, China). A total of 624 eligible Han Chinese participants (age, 40-65 years) were recruited. The Medical Ethics Committee of Sir Run Run Shaw Hospital affiliated to School of Medicine, Zhejiang University (Hangzhou, China) approved the present study. Written informed consent was obtained from the patients. Participants were $56.80 \pm 6.54$ years and $51.25 \%$ were male. All patients completed a population-based cross-sectional survey and were assigned a number. Random numbers were generated electronically and 40 patients with MetS (56.78 \pm 6.69 years; $45 \%$ male) and 40 control subjects $(56.83 \pm 6.00$ years; $57.5 \%$ male) were selected. Baseline anthropometric and metabolic measures were recorded using standardized methods $(19,20)$. Participants were interviewed face-to-face, completing a questionnaire regarding demographic data, life style, present and past illness, medical therapy and other health-associated information. All measurements were referred to as previously reported $(19,20)$.

Diagnosis of MetS. MetS was diagnosed according to criteria established by the Joint Committee for Developing Chinese Guidelines on Prevention and Treatment of Dyslipidemia in Adults (21). Individuals with $\geq 3$ of the following abnormalities were considered to have MetS: Central obesity [waist circumference (WC), $>90 \mathrm{~cm}$ for men and $>85 \mathrm{~cm}$ for women]; hypertriglyceridemia $(\geq 1.70 \mathrm{mmol} / \mathrm{l})$; high density lipoprotein-cholesterol (HDL-c; $<1.04 \mathrm{mmol} / \mathrm{l}$ ); blood pressure (BP; $\geq 130 / 85 \mathrm{mmHg}$ or ongoing treatment for hypertension); and hyperglycemia [fasting plasma glucose $(\mathrm{FPG}) \geq 6.1 \mathrm{mmol} / 1$ or $2 \mathrm{~h}$ postprandial glucose $\geq 7.8 \mathrm{mmol} / \mathrm{l}$ ]. Patients suffering from abnormal renal function or renal dysfunction at the time of recruitment were excluded. Patients fulfilling none of the above criteria were selected as healthy controls.

Patient data. Baseline anthropometric and metabolic measures were collected from all patients. Anthropometric data included height, weight, WC, hip circumference, heart rate and BP. The body mass index (BMI) was calculated as weight $(\mathrm{kg}) /[$ height $(\mathrm{m})]^{2}$. Systolic (S) BP and diastolic BP were calculated as the mean of three measurements, using a mercury sphygmomanometer at 3-min intervals. The body fat (\%) was assessed using a Tanita Body Composition Analyzer TBF-300 (Tanita Corporation, Tokyo, Japan). All subjects underwent abdominal magnetic resonance imaging (MRI) using a whole-body imaging system (SMT-100; Shimadzu Corporation, Kyoto, Japan) with TR-500 and TE-200 of spin-echo sequences (22). MRI scans were performed at the level of umbilicus between L4 and L5 with the subject in the supine position. Abdominal visceral fat area (VFA) and subcutaneous fat area (SFA) were calculated using Slice O'matic software (version 4.2; TomoVision, Magog, Canada). Following overnight fasting, blood and urine samples were collected; $5 \mathrm{ml}$ blood were stored in collection tubes with EDTA as an anticoagulant.

FPG, 2 h postprandial glucose, triglyceride (TG), total cholesterol, aspartate aminotransferase, alanine aminotransferase, low-density lipoprotein-cholesterol (LDL-c) and HDL-c were measured using an auto-analyzer (Abbott Pharmaceutical Co. Ltd., Lake Bluff, IL, USA). Fasting serum insulin levels (FINS), 2h postprandial insulin levels were measured by using an insulin detection kit (cat. no. 2400218; Beijing North Institute Biological Technology, Beijing, China). Insulin sensitivity was assessed by homeostasis model assessment for insulin resistance (HOMA-IR) based on FPG and insulin measurements as follows: [FINS (mU/l) x FPG (mmol/1) /22.5 (23). Glycosylated hemoglobin A1c (HbA1c) values were tested using ion-exchange high-performance liquid chromatography VARIANT II (D-100TM Hemoglobin Testing System; Bio-Rad Laboratories, Inc., Hercules, CA, USA) as described previously (24).

Urinary miRNA microarray. Genome-wide urinary miRNA profiles were detected using a microarray in whole urinary samples from 4 patients with MetS and 4 controls. Patients for the microarray analysis were selected at random out of the 40 patients previously identified per group. Comprehensive coverage was ensured using the TaqMan ${ }^{\circledR}$ Array Human MicroRNA Cards B v3 (cat. no. 4444910; Thermo Fisher Scientific, Inc., Waltham, MA, USA), resulting in a total of 377 unique assays specific for human miRNAs. A total of 8 RNA samples were analyzed by Kangchen BioTech Co., Ltd. (Shanghai, China) according to manufacturers' instructions.

Urinary miRNA detection. Total RNA was extracted from urine using miRNeasy kit (Qiagen Inc.) and purified using the Urine RNA Purification kit (Abnova, Taipei, Taiwan) according to manufacturers' instructions. For each reaction, $0.5-1 \mu \mathrm{g}$ of total RNA isolated from 5-10 ml of whole urine samples was used. MiDETECT A Track ${ }^{\mathrm{TM}}$ miRNA RT-PCR Start kit (R10048.3; Guangzhou RiboBio Co.,Ltd., Guangzhou, China) was used for validating miR-29a-3p and U6 expression via stem-loop-based reverse transcription-quantitative polymerase chain reaction (RT-qPCR) following the manufacturers' instructions and the following thermocycling conditions: $95^{\circ} \mathrm{C}$ for $5 \mathrm{~min}$ followed by 35 cycles of $95^{\circ} \mathrm{C}$ for $10 \mathrm{sec}, 60^{\circ} \mathrm{C}$ for $20 \mathrm{sec}$ and $70^{\circ} \mathrm{C}$ for 10 sec. miRNA expression was normalized to U6 using the $2^{-\Delta \Delta \mathrm{Cq}}$ method (25). Primer details are presented in Table I.

Cell culture and treatments. 293 cells were selected for the transfection experiments. Cells were purchased from the American Type Culture Collection (Manassas, VA, USA) and cultured in Dulbecco's modified Eagle's medium (DMEM; 
Table I. Polymerase chain reaction assays primers.

\begin{tabular}{ll}
\hline Gene & \multicolumn{1}{c}{ Primer sequences $\left(5^{\prime}-3^{\prime}\right)$} \\
\hline miR-29a-3p & \\
RT & GTCGTATCCAGTGCAGGGTCCGAGGTATTCGCACTGGATACGACTAACCG \\
qPCR forward & GCGCGTAGCACCATCTGAAAT \\
qPCR reverse & CAGTGCAGGGTCCGAGGT \\
U6 & \\
RT & TCGTATCCAGTGCAGGGTCCGAGGTATTCGCACTGGATACGACAAAATATG \\
qPCR forward & GCGCGTCGTGAAGCGTTC \\
qPCR reverse & CAGTGCAGGGTCCGAGGT
\end{tabular}

RT, reverse transcription; qPCR, quantitative polymerase chain reaction.

11965-092; Thermo Fisher Scientific, Inc.) containing $10 \%$ fetal bovine serum (FBS; Bio-Rad Laboratories, Inc.) and $100 \mathrm{IU} / \mathrm{ml}$ penicillin/streptomycin (Sigma-Aldrich; Merck KGaA, Darmstadt, Germany) at $37^{\circ} \mathrm{C}$ with $5 \% \mathrm{CO}_{2}$. Cholesterol-conjugated $2^{\prime}$ - $O$-methyl-modified mimics agomir-29a-3p (cat. no. miR40000210), agomir-NC (cat. no. miR04101), antagomir-29a-3p (cat. no. miR30000256) and antagomir-NC (cat. no. miR03101) were synthesized by Guangzhou RiboBio Co., Ltd. and used in transfection experiments. 293 cells were grown to $30-40 \%$ confluence and agomir-29a-3p (100 nM) or antagomir-29a-3p (200 nM) or respective controls were transiently transfected using riboFECTTM CP transfection kit (cat. no. R10035.3; RiboBio Co., Ltd, Guangzhou, China) according to the manufacturer's instructions (26). Cells were harvested at $48 \mathrm{~h}$ following treatment and RT-qPCR analysis was performed to assess transfection efficiency.

Dual-luciferase reporter assays. The homo sapiens insulin-like growth factor 1 (IGF1) 3'-untranslated region (3'UTR) was amplified from 293 cells and cloned into the XhoI/HindIII sites of the pGL4-basic vector (Promega Corporation, Madison, WI, USA). Cloning products were verified by sequencing and primer sequences were as follows: Forward, 5'-CCGCTCGAGACC ATCTCATGCTCTGTGGC-3' and reverse, 5'-CCCAAG CTTCTTTGCAAGGGAGGGGCATA-3'. 293 cells $\left(5 \times 10^{4}\right)$ were seeded in 24 -well plates $24 \mathrm{~h}$ prior to transfection. IGF1 plasmid (500 ng) and internal control Renilla luciferase plasmid (200 ng) were co-transfected using Lipofectamine 2000 (Invitrogen; Thermo Fisher Scientific, Inc.). Following 6-8 h, $100 \mathrm{nM}$ agomir-29a-3p and agomir-NC were transiently transfected into the cells using the riboFECTTM CP transfection kit. Cells were harvested at $48 \mathrm{~h}$ post-transfection and assayed for luciferase activity using the Dual-Luciferase Reporter Assay System (Promega Corporation). Activities were normalized to Renilla and all experiments were repeated four times.

RNA extraction and RT-qPCR. Total RNA was isolated from 293 cells using TRIzol reagent (Invitrogen; Thermo Fisher Scientific, Inc.) and RT was performed using a reverse transcription reagent kit (Takara Bio, Inc., Otsu, Japan) to generate the cDNA and samples were incubated for $30 \mathrm{~min}$ at $16^{\circ} \mathrm{C}$, followed by $30 \mathrm{~min}$ at $42^{\circ} \mathrm{C}$ and $5 \mathrm{~min}$ at $85^{\circ} \mathrm{C}$. qPCR was performed using a PCR kit containing SYBR Green (Takara Bio, Inc.) and a 7500 real-time PCR detection system (Applied Biosystems; Thermo Fisher Scientific, Inc.). Thermocycling conditions were as follows: $95^{\circ} \mathrm{C}$ for $30 \mathrm{sec}$ followed by 40 cycles of $95^{\circ} \mathrm{C}$ for $5 \mathrm{sec}, 60^{\circ} \mathrm{C}$ for $30 \mathrm{sec}$ and $70^{\circ} \mathrm{C}$ for $10 \mathrm{sec}$. IGF1 (forward, 5'-GGAGGCTGGAGATGTATTGC-3'and reverse, 5'-ACTTGCTTCTGTCCCCTCCT-3') expression was normalized to GAPDH (forward, 5'-AGCAGTCCCGTACAC TGGCAAAC-3'and reverse, 5'-TCTGTGGTGATGTAAAT GTCCTCT-3') and samples were quantified as described above. Measurements were conducted in triplicate.

Western blotting. Whole cell extracts were obtained by lysing cells in buffer $(50 \mathrm{mM}$ Tris pH 7.4, $150 \mathrm{mM} \mathrm{NaCl}, 1 \mathrm{mM}$ EDTA, $10 \mathrm{mM}$ sodium pyrophosphate, $0.5 \%$ Triton $\mathrm{X}-100$, $1 \mathrm{mM}$ sodium vanadate and $25 \mathrm{mM}$ sodium fluoride) containing protease inhibitors $(5 \mu \mathrm{g} / \mathrm{ml}$ phenylmethylsulfonyl fluoride, $0.5 \mu \mathrm{g} / \mathrm{ml}$ leupeptin, $0.7 \mu \mathrm{g} / \mathrm{ml}$ pepstatin and $0.5 \mu \mathrm{g} / \mathrm{ml}$ aprotinin). Protein concentrations were determined using a bicinchoninic acid protein assay. Equal amounts of protein $(50 \mu \mathrm{g})$ were denatured by boiling, separated using $10 \%$ SDS-PAGE gels and transferred to polyvinylidene membranes. Membranes were blocked with 5\% non-fat milk for $1 \mathrm{~h}$ at room temperature. Membranes were then incubated with primary antibodies, including polyclonal rabbit anti-IGF1 (cat. no. ab40657; 1:1,000; Abcam, Cambridge, UK) and monoclonal mouse anti- $\beta$-actin (cat. no. A5316; 1:5,000; Sigma-Aldrich; Merck KGaA) at $4^{\circ} \mathrm{C}$ overnight followed by incubation with horseradish peroxidase-conjugated goat anti-rabbit/mouse secondary IgG antibodies (cat. nos. ab6721 and ab6789; 1:10,000; Abcam) at room temperature for $1 \mathrm{~h}$. Immunoreactive proteins were detected using a chemiluminescent assay kit (EMD Millipore, Billerica, MA, USA). Quantity One software (v4.6.2; Bio-Rad Laboratories, Inc.) was used to quantify expression levels and normalize to $\beta$-actin.

Bioinformatics databases. To search for the enrichment of specific target genes associated with insulin signaling and the glucose and lipid metabolism, the Database for Annotation, Visualization and Integrated Discovery (https://david.ncifcrf. gov) were used. To predict target genes of miR-29a-3p, bioinformatics databases miRanda (http://www.microrna.org), 
Table II. Clinical characteristics of the participants included in the validation study.

\begin{tabular}{|c|c|c|c|c|}
\hline Characteristic & Total & Control & MetS & P-value \\
\hline $\mathrm{N}$ & 80 & 40 & 40 & \\
\hline Age (years) & $56.80 \pm 6.54$ & $56.83 \pm 6.00$ & $56.78 \pm 6.69$ & 0.743 \\
\hline Male, $\mathrm{n}(\%)$ & $41(51.25)$ & $18(45.00)$ & $23(57.50)$ & 0.532 \\
\hline Current smoker, n(\%) & $34(42.5)$ & $8(10)$ & $26(32.5)$ & $<0.001$ \\
\hline Alcohol drinker, n(\%) & $25(31.25)$ & $13(16.25)$ & $12(15)$ & 0.117 \\
\hline BMI $\left(\mathrm{kg} / \mathrm{m}^{2}\right)$ & $23.78 \pm 3.04$ & $21.99 \pm 2.24$ & $25.28 \pm 2.50$ & $<0.001$ \\
\hline WC (cm) & $80.54(68.13-91.00)$ & $73.00(68.13-83.00)$ & $86.50(83.25-91.00)$ & $<0.001$ \\
\hline WHR & $0.88(0.80-0.97)$ & $0.85(0.80-0.89)$ & $0.92(0.89-0.97)$ & $<0.001$ \\
\hline Body fat (\%) & $28.68 \pm 6.47$ & $26.26 \pm 5.65$ & $30.87 \pm 6.39$ & 0.001 \\
\hline $\mathrm{SBP}(\mathrm{mmHg})$ & $123.41(110.00-141.42)$ & $116.67(110.00-123.30)$ & 124.67(118.84-141.42) & $<0.001$ \\
\hline DBP (mmHg) & $81.34 \pm 9.21$ & $78.32 \pm 7.88$ & $84.10 \pm 8.71$ & $<0.001$ \\
\hline HbA1c $(\%)$ & $5.65(5.30-6.00)$ & $5.50(5.13-5.70)$ & $5.70(5.40-6.20)$ & 0.012 \\
\hline ALT (U/l) & $18.00(14.00-26.00)$ & $17.70(10.90-25.50)$ & $29.10(16.90-33.30)$ & 0.001 \\
\hline AST (U/l) & $19.00(16.00-23.00)$ & $19.70(14.70-24.70)$ & $23.50(17.50-29.50)$ & 0.174 \\
\hline FPG (mmol/l) & $5.23(4.60-5.52)$ & $6.71(6.44-7.40)$ & $7.42(6.85-7.81)$ & 0.002 \\
\hline $2 \mathrm{~h}$ postprandial glucose $(\mathrm{mmol} / \mathrm{l})$ & $6.03(4.82-7.11)$ & $7.58(6.4-8.4)$ & $10.17(7.65-15.26)$ & $<0.001$ \\
\hline FINS $(\mu \mathrm{U} / \mathrm{ml})$ & $10.66(8.18-13.87)$ & $11.48 \pm 3.18$ & $18.23 \pm 4.01$ & $<0.001$ \\
\hline $2 \mathrm{~h}$ postprandial insulin $(\mu \mathrm{U} / \mathrm{ml})$ & $57.25(37.32-87.80)$ & $54.35(36.39-83.57)$ & $81.68(47.75-155.18)$ & $<0.001$ \\
\hline HOMA-IR & $2.77(1.73-3.13)$ & $2.10(1.68-2.87)$ & $3.52(2.55-5.26)$ & $<0.001$ \\
\hline $\mathrm{TC}(\mathrm{mmol} / \mathrm{l})$ & $5.52 \pm 1.02$ & $5.37 \pm 0.88$ & $5.74 \pm 1.07$ & 0.301 \\
\hline LDL-c (mmol/l) & $2.25(2.00-2.64)$ & $2.34(1.90-2.78)$ & $2.29(1.71-2.87)$ & 0.807 \\
\hline HDL-c (mmol/l) & $1.31(1.03-1.66)$ & $1.65(1.35-1.95)$ & $1.10(0.86-1.34)$ & $<0.001$ \\
\hline TG $(\mathrm{mmol} / \mathrm{l})$ & $1.57(0.88-2.21)$ & $1.03(0.61-1.45)$ & $3.23(1.81-3.26)$ & $<0.001$ \\
\hline $\mathrm{SFA}\left(\mathrm{cm}^{2}\right)$ & $152.70(122.53-192.35)$ & $150.68(105.75-205.05)$ & $170.43(122.25-228.14)$ & $<0.001$ \\
\hline $\operatorname{VFA}\left(\mathrm{cm}^{2}\right)$ & $70.58(47.18-122.17)$ & $55.47(23.23-87.53)$ & $118.24(72.22-164.34)$ & $<0.001$ \\
\hline
\end{tabular}

Data are presented as the mean \pm standard deviation, the median with $25-75 \%$ interquartile range or $\mathrm{n}(\%)$. BMI, body mass index; WC, waist circumference; WHR, waist-to-hip ratio; SBP, systolic blood pressure; DBP, diastolic blood pressure; HbA1c, hemoglobin A1c; ALT, alanine transaminase; AST, aspartate transaminase; FPG, fasting plasma glucose; FINS, fasting serum insulin; HOMA-IR, homeostasis model assessment-insulin resistance; TC, total cholesterol; LDL-c, low-density lipoprotein-cholesterol; HDL-c high density lipoprotein-cholesterol; TG, triglyceride; SFA, abdominal subcutaneous fat area; VFA, visceral fat area.

mirBase (http://www.mirbase.org) and Targetscan (http:// www.targetscan.org) were used.

Statistical analysis. For the patient study, all the continuous variables were tested for normal distribution and normally distributed variables are expressed as the mean \pm standard deviation and variables with a skewed distribution are presented as the median with $25-75 \%$ interquartile range. Categorical variables were presented as frequencies and percentages. Differences in baseline characteristics were analyzed by two-sided Student's t-test for continuous variables and chi-square test for categorical variables. Correlation between urinary miR-29a-3p and metabolic parameters was determined using a Spearman correlation analysis. MetS incidence at different levels of urinary miR-29a-3p was analyzed by chi-square test. Logistic regression analysis and multiple stepwise regression analysis were applied in the analysis of the adjusted variables. A receiver operating characteristic (ROC) analysis was conducted and areas under the curve and optimal sensitivity and specificity levels were calculated. SPSS 17.0 (SPSS, Inc., Chicago, IL, USA) was used for statistical analyses.
Cell experiments were evaluated using independent two-sided Student's t-test and one-way analysis of variance followed by Tukey's test for pairwise or multiple group comparisons, respectively. Data are presented as the mean \pm standard deviation, representative of three replicates. $\mathrm{P}<0.05$ was considered to indicate a statistically significant difference.

\section{Results}

Baseline patient characteristics. Anthropometric and metabolic characteristics of the study population at baseline are presented in Table II. Participants with MetS exhibited increased cardiovascular risk factors compared with the controls, including significantly higher BMI, waist-to-hip ratio, body fat, FINS levels, HOMA-IR, HbA1c, SFA, VFA, and MetS indicators, including significantly higher WC, blood glucose, TG, BP and significantly lower HDL-c $(\mathrm{P}<0.05)$.

Microarray analysis reveals that urinary miR-29a-3p is upregulated in patients with MetS. Urine samples from 4 patients with MetS and 4 controls were analyzed using a 
A

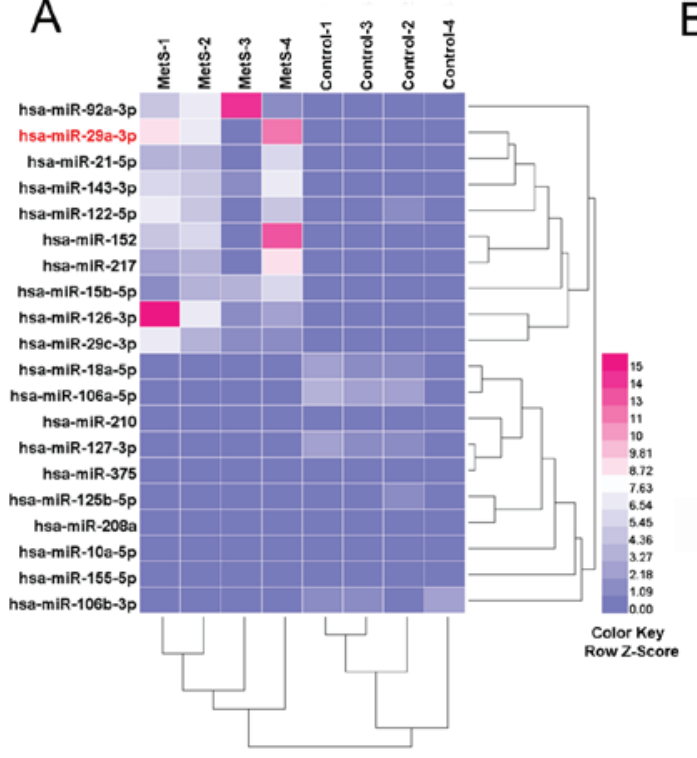

B

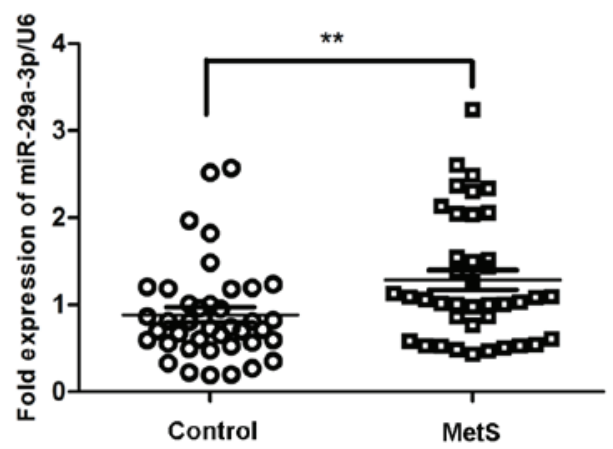

Figure 1. miR-29a-3p expression in patients with MetS. (A) Heat map of the top 20 differentially expressed miRNAs as evaluated by microarray using urine samples from patients with MetS and control subjects ( $n=4 /$ group). (B) miR-29a-3p expression determined by reverse transcription-quantitative polymerase chain reaction analysis using urinary samples from patients with MetS and controls ( $\mathrm{n}=40 /$ group). ${ }^{* *} \mathrm{P}<0.01$. MetS, metabolic syndrome; miR, microRNA.

microarray evaluating a total of 377 unique assays specific to human miRNAs. Among the 377 screened miRNAs, 20 were identified as differentially expressed in the urine of patients with MetS compared with the controls (fold change $>2$; $\mathrm{P}<0.05$; Fig. $1 \mathrm{~A})$. Based on the microarray analysis, miR-29a-3p was selected for further validation by RT-qPCR using a larger sample size ( $n=40 /$ group). RT-qPCR analyses confirmed that urinary miR-29a-3p levels were significantly increased in patients with MetS compared with the controls $(\mathrm{P}<0.01$; Fig. 1B). Furthermore, participants with MetS had significantly increased urinary miR-29a-3p levels compared with the control $(0.51 \pm 0.17$ vs. $0.34 \pm 0.20 ; \mathrm{P}<0.01)$.

Correlation between urinary miR-29a-3p and metabolic parameters. Associations between urinary miR-29a-3p levels and parameters associated to adiposity, insulin resistance, lipid profiles and hepatic enzymes were further assessed. Spearman correlation analyses of urinary miR-29a-3p with metabolic risks were performed. As presented in Table III, following an adjustment for gender, age, smoking and drinking, urinary miR-29a-3p levels were correlated with HOMA-IR $(r=0.2713$; $\mathrm{P}<0.001)$, FINS $(\mathrm{r}=0.643 ; \mathrm{P}<0.001)$ and HDL-c $(\mathrm{r}=0.242$; $\mathrm{P}<0.001$ ). Additionally, urinary miR-29a-3p levels were correlated with BMI, SBP, FPG and body fat ( $\mathrm{p}<0.05$; Table III).

Urinary miR-29a-3p levels are independently associated with FINS, HDL-c and BMI. Furthermore, it was assessed whether a high level of urinary miR-29a-3p may be used to predict the incidence of further metabolism subgroups, including central obesity, hypertension, hyperglycemia, dyslipidemia, and low HDL-c in patients with MetS. Multiple stepwise regression analysis using urinary miR-29a-3p levels as the dependent variable following the adjustment for gender, age, smoking and drinking revealed that urinary miR-29a-3p levels were independently associated with FINS $(\beta=0.561 ; \mathrm{P}<0.001)$, HDL-c $(\beta=0.242 ; \mathrm{P}<0.001)$ and $\mathrm{BMI}(\beta=-0.141 ; \mathrm{P}<0.05$; Table IV $)$.

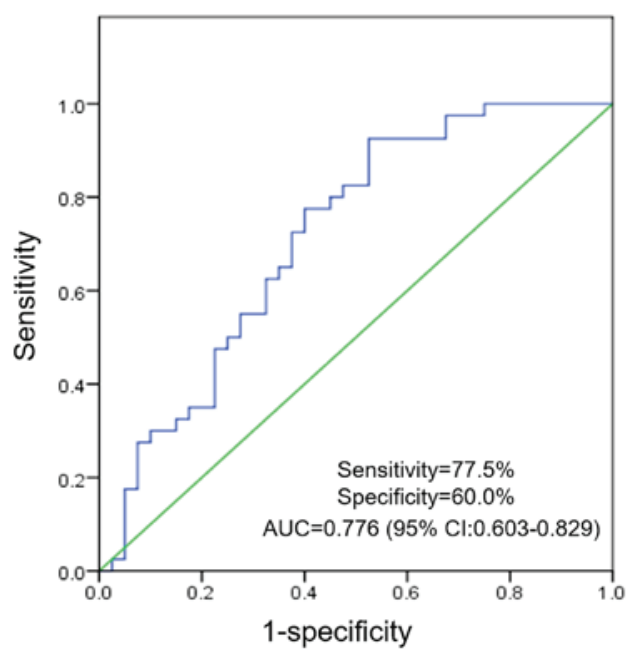

Figure 2. Urinary miR-29a-3p levels allow distinguishing between patients with MetS and controls. Receiver operating characteristic curve analysis compared miR-29a-3p levels of urine samples from patients with MetS and controls. MetS, metabolic syndrome; miR, microRNA; AUC, area under the curve; CI, confidence interval.

Urinary miR-29a-3p levels have a diagnostic value for MetS. To assess the accuracy and clinical utility of urinary miR-29a-3p levels in differentiating between patients with MetS and control subjects in the validation phase ( $n=40$ /group), a ROC curve analysis was performed using urinary miR-29a-3p levels. Using a logistic regression model, analysis indicated that urinary miR-29a-3p may be a valuable biomarker in patient with MetS compared with healthy controls, with an area under the curve of 0.776 (95\% CI, 0.603-0.829). The cut-off value for urinary miR-29a-3p levels was 0.375 and the optimal sensitivity and specificity were 77.5 and 60.0\%, respectively (Fig. 2).

IGF1 is a target gene of miR-29a-3p. To identify potential molecular targets of miR-29a-3p in patients with MetS, 
Table III. Spearman correlation analyses of urinary microRNA-29a-3p with metabolic parameters prior to and following age-, sex-, smoking- and drinking-adjustments.

\begin{tabular}{|c|c|c|c|c|}
\hline \multirow[b]{2}{*}{ Parameter } & \multicolumn{2}{|c|}{ Unadjusted } & \multicolumn{2}{|c|}{$\begin{array}{l}\text { Age, sex, smoking } \\
\text { and drinking-adjusted }\end{array}$} \\
\hline & $\mathrm{r}$ & P-value & $\mathrm{r}$ & P-value \\
\hline BMI $\left(\mathrm{kg} / \mathrm{m}^{2}\right)$ & 0.334 & 0.002 & 0.345 & 0.002 \\
\hline $\mathrm{WC}(\mathrm{cm})$ & 0.250 & 0.025 & 0.271 & 0.018 \\
\hline WHR & 0.147 & 0.193 & 0.212 & 0.066 \\
\hline Body fat (\%) & 0.302 & 0.009 & 0.354 & 0.008 \\
\hline $\mathrm{SBP}(\mathrm{mmHg})$ & 0.331 & 0.003 & 0.370 & 0.001 \\
\hline $\mathrm{DBP}(\mathrm{mmHg})$ & 0.098 & 0.386 & 0.087 & 0.457 \\
\hline $\operatorname{ALT}(\mathrm{U} / \mathrm{l})$ & 0.023 & 0.818 & 0.047 & 0.653 \\
\hline $\operatorname{AST}(\mathrm{U} / \mathrm{l})$ & -0.002 & 0.980 & -0.009 & 0.929 \\
\hline FPG (mmol/l) & 0.234 & 0.037 & 0.277 & 0.015 \\
\hline $2 \mathrm{~h}$ postprandial glucose $(\mathrm{mmol} / \mathrm{l})$ & 0.001 & 0.993 & 0.153 & 0.187 \\
\hline FINS $(\mu \mathrm{U} / \mathrm{ml})$ & 0.624 & $<0.001$ & 0.634 & $<0.001$ \\
\hline $2 \mathrm{~h}$ postprandial insulin $(\mu \mathrm{U} / \mathrm{ml})$ & 0.307 & 0.006 & 0.275 & 0.056 \\
\hline HOMA-IR & 0.707 & $<0.001$ & 0.726 & $<0.001$ \\
\hline $\mathrm{HbA} 1 \mathrm{C}(\%)$ & 0.193 & 0.086 & 0.175 & 0.130 \\
\hline $\mathrm{TC}(\mathrm{mmol} / \mathrm{l})$ & 0.054 & 0.633 & 0.014 & 0.905 \\
\hline LDL-c (mmol/l) & 0.132 & 0.242 & 0.042 & 0.718 \\
\hline HDL-c (mmol/l) & -0.437 & $<0.001$ & -0.497 & $<0.001$ \\
\hline TG (mmol/l) & 0.377 & $<0.001$ & 0.042 & 0.721 \\
\hline SFA $\left(\mathrm{cm}^{2}\right)$ & 0.189 & 0.093 & 0.226 & 0.050 \\
\hline $\operatorname{VFA}\left(\mathrm{cm}^{2}\right)$ & 0.287 & 0.010 & 0.272 & 0.017 \\
\hline
\end{tabular}

BMI, body mass index; WC, waist circumference; WHR, waist-to-hip ratio; SBP, systolic blood pressure; DBP, diastolic blood pressure; HbA1c, hemoglobin A1c; ALT, alanine transaminase; AST, aspartate transaminase; FPG, fasting plasma glucose; FINS, fasting serum insulin; HOMA-IR, homeostasis model assessment-insulin resistance; TC, total cholesterol; LDL-c, low-density lipoprotein-cholesterol; HDL-c high density lipoprotein-cholesterol; TG, triglyceride; SFA, abdominal subcutaneous fat area; VFA, visceral fat area.

Table IV. Multiple stepwise regression analyses of independent factors associated with urinary microRNA-29a-3p levels.

\begin{tabular}{lcc}
\hline Variable & $\beta$ & P-value \\
\hline FINS & 0.561 & $<0.001$ \\
HDL-c & 0.245 & 0.001 \\
BMI & -0.141 & 0.025 \\
\hline
\end{tabular}

BMI, body mass index; FINS, fasting insulin; HDL-c high density lipoprotein-cholesterol.

gene ontology and biological association analyses using the Database for Annotation, Visualization and Integrated Discovery Bioinformatics Resources were performed (27) and searched for enrichment of specific target genes associated with insulin signaling, as well as glucose and lipid metabolism. Following evaluation of three widely used bioinformatics databases (20), it was determined that IGF1 was a potential target of miR-29a-3p (Fig. 3A).
Subsequently, a dual-reporter constructs was generated by fusion of the luciferase coding sequence to the 3'UTR of IGF1. Luciferase activity of the reporter constructs containing the predicted miR-29a-3p binding sites was determined. The dual-reporter luciferase assay revealed that agomir-29a-3p treatment significantly repressed luciferase activity of IGF1 compared with the control (48.97\% reduction; $\mathrm{P}<0.01$; Fig. $3 \mathrm{~B}$ ) and agomir-NC treatment had no significant effect. The results suggested that IGF1 was a direct target of miR-29a-3p.

To investigate whether miR-29a-3p expression effects to IGF1 levels, 293 cells were treated with miR-29a-3p agomir and antagomir for upregulation and knockdown of miR-29a-3p, respectively. Agomir-29a-3p significantly upregulated miR-29a-3p levels compared with the agomir-NC treated cells (7.24-fold increase; $P<0.001$; Fig. 3C). Antagomir-29a-3p treatment significantly reduced miR-29a-3p levels compared with antagomir-NC treated cells $(\sim 70 \%$ reduction; $\mathrm{P}<0.01$; Fig. 3C). mRNA expression of IGF1was assessed following miR-29a-3p agomirs and antagomirs treatment. Compared with the negative controls, IGF1 levels were significantly reduced by agomir-29a-3p $(\mathrm{P}<0.05)$ and significantly increased by antagomir-29a-3p treatment ( $\mathrm{P}<0.01$; Fig. $3 \mathrm{C})$. Results were confirmed by western blot analysis, exhibiting 
A

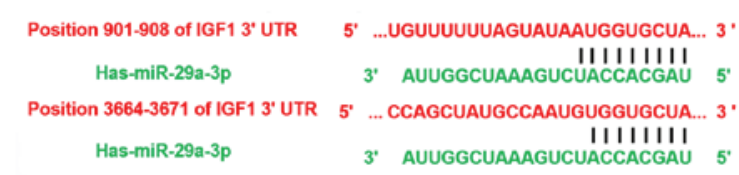

B

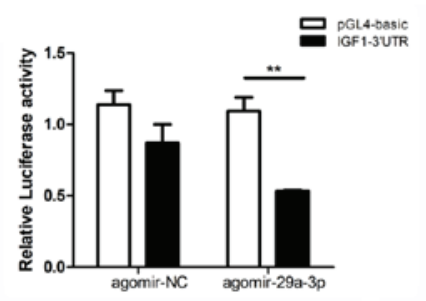

D

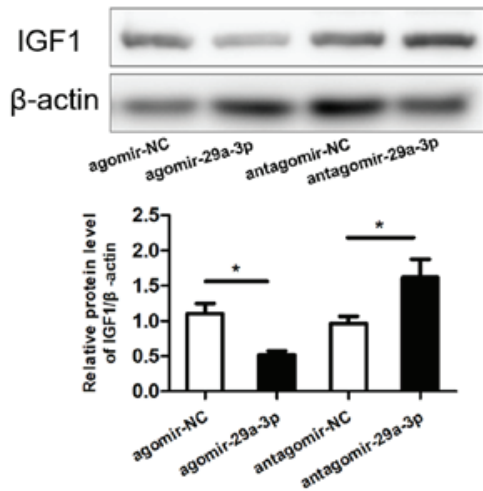

Figure 3. IGF1 is a target gene of miR-29a-3p. (A) Predicted binding of miR-29a-3p to IGF1 based on Miranda, Mirbase and Targetscan. (B) Luciferase reporter assay activity determined in 293 cells; results represent three independent experiments. (C) miR-29a-3p and IGF1 mRNA expression in agomir and antagomir treated 293 cells determined by reverse transcription-quantitative polymerase chain reaction; results represent three independent experiments. (D) Western blot analysis of IGF1 expression in agomir and antagomir treated 293 cells; experiments were repeated three times. ${ }^{*} \mathrm{P}<0.05$, $^{* *} \mathrm{P}<0.01$ and ${ }^{* * * *} \mathrm{P}<0.001$. IGF1, insulin-like growth factor 1 ; miR, microRNA; NC, negative control.

that protein levels of IGF1 were significantly downregulated by agomir-29a-3p and upregulated by antagomir-29a-3p compared with the controls $(\mathrm{P}<0.05$; Fig. 3D).

\section{Discussion}

The diagnostic role of urinary miRNAs has been successfully explored in several conditions, including type 2 diabetic kidney disease $(9,28,29)$. A meta-analysis revealed that urinary and blood miR-126 and miR-770 are potential noninvasive biomarker candidates for diabetic nephropathy (30). Deli et al (28) suggested that in patients with type 2 diabetic nephropathy the urinary exosomal miRNA signature of miR-320c may function as a novel marker. Peng et al (31) revealed urinary miR-29 as a novel biomarker for diabetic nephropathy and atherosclerosis in patients with type 2 diabetes. Liu et al (32) observed that miR-29b was upregulated in the renal medulla of Dahl salt-sensitive (SS) rats and that miR-29b affects numerous collagens and genes associated with the extracellular matrix, suggesting an involvement of miR-29b in the protection from renal medullary injury in SS rats. Here, a microarray analysis revealed that urine levels of miR-29a-3p were significantly increased in patients with MetS compared with controls ( $\mathrm{n}=4$ /group) and results were validated using RT-qPCR analysis in a larger cohort ( $n=40 /$ group). Urinary miR-29a-3p levels were further correlated with independent parameters associated with the metabolism, including FINS, HDL-c and BMI.

In addition to using urinary miRNAs as potential clinical biomarker, Zavesky et al (33) demonstrated urinary miRNA expression analysis has potential in identifying novel diagnostic and prognostic markers. Particularly due to stability and accessible nature, urinary miRNAs may be used as noninvasive biomarkers for graft injury. Using urinary miRNA expression levels as diagnostic tools remains challenging and the process is still in its infancy regarding detection techniques and costs $(7,34,35)$. In the current study, ROC curve analysis assessed the accuracy and clinical applicability of evaluating urinary miR-29a-3p levels in patients with MetS. Elevated urinary miR-29a-3p levels were positively associated with MetS and may have a predictive value as biomarkers in the diagnosis of MetS.

The current study had several limitations. Urinary miRNA levels were detected in whole urine samples, raising the question whether whole urine samples should be used. Due to the unclear origin of the miRNAs detected in the urine $(35,36)$, results need to be regarded with caution. Until now, numerous studies investigating urinary miRNA levels used urinary cell pellets; however, no consistent procedure for obtaining these pellets was followed $(37,38)$. To avoid contamination with cells from the lower urinary tract, midstream urine samples function as sources in biomarker analysis for kidney pathology (7). In the current study, patients that suffered from abnormal renal function or renal dysfunction were excluded; however, it was not accounted for other inflammatory or autoimmune disorders at the time of recruitment.

The present study identified and confirmed IGF1 as a target of miR-29a-3p. Ligands of the IGF system, including insulin, IGF1 and IGF2, exert their biological effects by binding to the insulin receptor (39). Previous research revealed that IGF1 levels are associated with the prevalence of MetS and increases in triglyceride levels in elderly patients $(40,41)$. Decreased IGF1 levels are associated with increased metabolic burden $(42,43)$. The findings of the current study 
provided evidence for miR-29a-3p involvement in regulating IGF1 expression in a 293 cell model and as no patient-derived cells were utilized conclusions may not be applicable and/or validated in in vivo settings.

In summary, the presented study suggested an association between urinary miR-29a-3p and IGF1 in patients with MetS. Urinary miR-29a-3p may accelerate obesity-induced insulin resistance, glucose tolerance and lipid accumulation via regulation of the IGF1. These findings further suggested that urinary miR-29a-3p may have potential as novel targets in the treatment of MetS. Elevated urinary miR-29a-3p levels were positively correlated with MetS and may be considered as predictive biomarkers in diagnosis of MetS.

\section{Acknowledgements}

Not applicable.

\section{Funding}

This project was supported by the Zhejiang Provincial Medical Science and Technology Program (grant nos. 2015DTA009, 2013RCA028 and 2018KY484), the National Science and Technology Support Program (grant no. 2009BAI80B02) and the National Natural Science Foundation of China (grant no. 81600667).

\section{Availability of data and materials}

The datasets used and/or analyzed during the current study are available from the corresponding author on reasonable request.

\section{Authors' contributions}

XHL and JQZ contributed to the conception and design of the study. CL, DJH and HL coordinated the study. XHL, DJH, EM, QLC and CL were involved in data acquisition and interpretation. XHL and EM drafted the manuscript. All authors read and approved the final manuscript.

\section{Ethics approval and consent to participate}

The Medical Ethics Committee of Sir Run Run Shaw Hospital affiliated to School of Medicine, Zhejiang University (Hangzhou, China) approved the current study.

\section{Patient consent for publication}

Not applicable.

\section{Competing interests}

The authors declared that they have no competing interests.

\section{References}

1. Shab-Bidar S, Golzarand M, Hajimohammadi M and Mansouri S A posteriori dietary patterns and metabolic syndrome in adults: A systematic review and meta-analysis of observational studies. Public Health Nutr 21: 1681-1692, 2018.

2. Krützfeldt J and Stoffel M: MicroRNAs: A new class of regulatory genes affecting metabolism. Cell Metab 4: 9-12, 2006.
3. Dehwah MA, Xu A and Huang Q: MicroRNAs and type 2 diabetes/obesity. J Genet Genomics 39: 11-18, 2012.

4. Carreras-Badosa G, Bonmatí A, Ortega FJ, Mercader JM, Guindo-Martínez M, Torrents D, Prats-Puig A, Martinez-Calcerrada JM, Platero-Gutierrez E, De Zegher F, et al: Altered Circulating miRNA Expression Profile in Pregestational and Gestational Obesity. J Clin Endocrinol Metab 100: E1446-E1456, 2015.

5. Liang Z, Gao KP, Wang YX, Liu ZC, Tian L, Yang XZ, Ding JY, Wu WT, Yang WH, Li YL, et al: RNA sequencing identified specific circulating miRNA biomarkers for early detection of diabetes retinopathy. Am J Physiol Endocrinol Metab 315: E374-E385, 2018.

6. Rayner KJ and Hennessy EJ: Extracellular communication via microRNA: Lipid particles have a new message. J Lipid Res 54: 1174-1181, 2013

7. van de Vrie M, Deegens JK, Eikmans M, van der Vlag J and Hilbrands LB: Urinary MicroRNA as Biomarker in Renal Transplantation. Am J Transplant 17: 1160-1166, 2017.

8. Endzelinš E, Berger A, Melne V, Bajo-Santos C, Sobolevska K, Ābols A,'RodriguezM,ŚStareD,RudnickihaA,Lietuvietis V,etal: Detection of circulating miRNAs: Comparative analysis of extracellular vesicle-incorporated miRNAs and cell-free miRNAs in whole plasma of prostate cancer patients. BMC Cancer 17: 730, 2017.

9. Xie Y, Jia Y, Xie C, Hu F, Xue M and Xue Y: Corrigendum to Urinary Exosomal MicroRNA Profiling in Incipient Type 2 Diabetic Kidney Disease. J Diabetes Res 2018: 5969714, 2018.

10. Mitchell PS, Parkin RK, Kroh EM, Fritz BR, Wyman SK, Pogosova-Agadjanyan EL, Peterson A, Noteboom J, O'Briant KC, Allen A, et al: Circulating microRNAs as stable blood-based markers for cancer detection. Proc Natl Acad Sci USA 105: 10513-10518, 2008.

11. Redova M, Sana J and Slaby O: Circulating miRNAs as new blood-based biomarkers for solid cancers. Future Oncol 9: 387-402, 2013.

12. He A, Zhu L, Gupta N, Chang Y and Fang F: Overexpression of micro ribonucleic acid 29 , highly up-regulated in diabetic rats, leads to insulin resistance in 3T3-L1 adipocytes. Mol Endocrinol 21: 2785-2794, 2007.

13. Song H, Ding L, Zhang S and Wang W: miR-29 family members interact with SPARC to regulate glucose metabolism. Biochem Biophys Res Commun 497: 667-674, 2018.

14. Bagge A, Clausen TR, Larsen S, Ladefoged M, Rosenstierne MW, Larsen L, Vang O, Nielsen JH and Dalgaard LT: MicroRNA-29a is up-regulated in beta-cells by glucose and decreases glucose-stimulated insulin secretion. Biochem Biophys Res Commun 426: 266-272, 2012.

15. Herrera BM, Lockstone HE, Taylor JM, Wills QF, Kaisaki PJ, Barrett A, Camps C, Fernandez C, Ragoussis J, Gauguier D, et al: MicroRNA-125a is over-expressed in insulin target tissues in a spontaneous rat model of Type 2 Diabetes. BMC Med Genomics 2: 54, 2009.

16. Pandey AK, Verma G, Vig S, Srivastava S, Srivastava AK and Datta M: miR-29a levels are elevated in the $\mathrm{db} / \mathrm{db}$ mice liver and its overexpression leads to attenuation of insulin action on PEPCK gene expression in HepG2 cells. Mol Cell Endocrinol 332: 125-133, 2011.

17. Liang J, Liu C, Qiao A, Cui Y, Zhang H, Cui A, Zhang S, Yang Y, Xiao X, Chen Y, et al: MicroRNA-29a-c decrease fasting blood glucose levels by negatively regulating hepatic gluconeogenesis. J Hepatol 58: 535-542, 2013.

18. Ślusarz A and Pulakat L: The two faces of miR-29. J Cardiovasc Med (Hagerstown) 16: 480-490, 2015.

19. Xueyao Y, Saifei Z, Dan Y, Qianqian P, Xuehong D, Jiaqiang Z, Fenping $\mathrm{Z}$ and Hong L: Circulating fractalkine levels predict the development of the metabolic syndrome. Int J Endocrinol 2014: 715148, 2014.

20. Xihua L, Shengjie T, Weiwei G, Matro E, Tingting T, Lin L, Fang W, Jiaqiang Z, Fenping Z and Hong L: Circulating miR 1433 p inhibition protects against insulin resistance in Metabolic Syndrome via targeting of the insulin like growth factor 2 receptor. Transl Res, 2018.

21. Chinese guidelines on prevention and treatment of dyslipidemia in adults. Zhonghua Xin Xue Guan Bing Za Zhi 35: 390 419, 2007 (In Chinese)

22. Yin XY, Zheng FP, Zhou JQ, Du Y, Pan QQ, Zhang SF, Yu D and Li H: Central obesity and metabolic risk factors in middle-aged Chinese. Biomed Environ Sci 27: 343-352, 2014. 
23. Emoto M, Nishizawa Y, Maekawa K, Hiura Y, Kanda H, Kawagishi T, Shoji T, Okuno Y and Morii H: Homeostasis model assessment as a clinical index of insulin resistance in type 2 diabetic patients treated with sulfonylureas. Diabetes Care 22: 818-822, 1999.

24. Jaisson S, Leroy N, Guillard E, Desmons A and Gillery P: Analytical performances of the D-100TM hemoglobin testing system (Bio-Rad) for HbA1c assay. Clin Chem Lab Med 53: 1473-1479, 2015

25. Liu S, Li W, Xu M, Huang H, Wang J and Chen X: Micro-RNA 21Targets dual specific phosphatase 8 to promote collagen synthesis in high glucose-treated primary cardiac fibroblasts. Can J Cardiol 30: 1689-1699, 2014.

26. Hu XX, Xu XN, He BS, Sun HL, Xu T, Liu XX, Chen XX, Zeng KX, Wang SK and Pan YQ: MicroRNA-485-5p Functions as a Tumor Suppressor in Colorectal Cancer Cells by Targeting CD147. J Cancer 9: 2603-2611, 2018.

27. Dennis G Jr, Sherman BT, Hosack DA, Yang J, Gao W, Lane HC and Lempicki RA: DAVID: Database for Annotation, Visualization, and Integrated Discovery. Genome Biol 4: 3, 2003.

28. Delić D, Eisele C, Schmid R, Baum P, Wiech F, Gerl M Zimdahl H, Pullen SS and Urquhart R: Urinary Exosomal miRNA Signature in Type II Diabetic Nephropathy Patients. PLoS One 11: e0150154, 2016.

29. Eissa S, Matboli M, Aboushahba R, Bekhet MM and Soliman Y: Urinary exosomal microRNA panel unravels novel biomarkers for diagnosis of type 2 diabetic kidney disease. J Diabetes Complications 30: 1585-1592, 2016.

30. Park S, Moon S, Lee K, Park IB, Lee DH and Nam S Urinary and Blood MicroRNA-126 and -770 are Potential Noninvasive Biomarker Candidates for Diabetic Nephropathy: A Meta-Analysis. Cell Physiol Biochem 46: 1331-1340, 2018.

31. Peng H, Zhong M, Zhao W, Wang C, Zhang J, Liu X, Li Y, Paudel SD, Wang Q and Lou T: Urinary miR-29 correlates with albuminuria and carotid intima-media thickness in type 2 diabetes patients. PLoS One 8: e82607, 2013.

32. Liu Y, Taylor NE, Lu L, Usa K, Cowley AW Jr, Ferreri NR, Yeo NC and Liang M: Renal medullary microRNAs in Dahl salt-sensitive rats: miR-29b regulates several collagens and related genes. Hypertension 55: 974-982, 2010.

33. Zavesky L, Jandakova E, Turyna R, Langmeierova L, Weinberger V, Minar L and Kohoutova M: New perspectives in diagnosis of gynaecological cancers: Emerging role of circulating microRNAs as novel biomarkers. Neoplasma 62: 509-520, 2015.
34. Campion CG, Sanchez-Ferras O and Batchu SN: Potential Role of Serum and Urinary Biomarkers in Diagnosis and Prognosis of Diabetic Nephropathy. Can J Kidney Health Dis 4: 2054358117705371, 2017

35. Mall C, Rocke DM, Durbin-Johnson B and Weiss RH: Stability of miRNA in human urine supports its biomarker potential. Biomarkers Med 7: 623-631, 2013.

36. Ben-Dov IZ, Tan YC, Morozov P, Wilson PD, Rennert H, Blumenfeld JD and Tuschl T: Urine microRNA as potential biomarkers of autosomal dominant polycystic kidney disease progression: Description of miRNA profiles at baseline. PLoS One 9: e86856, 2014.

37. Abulaban KM, Fall N, Nunna R, Ying J, Devarajan P, Grom A, Bennett M, Ardoin SP and Brunner HI: Relationship of cell-free urine MicroRNA with lupus nephritis in children. Pediatr Rheumatol Online J 14: 4, 2016.

38. Lv LL, Cao Y, Liu D, Xu M, Liu H, Tang RN, Ma KL and Liu BC: Isolation and quantification of microRNAs from urinary exosomes/microvesicles for biomarker discovery. Int J Biol Sci 9: 1021-1031, 2013

39. Lelbach A, Muzes G and Feher J: The insulin-like growth factor system: IGFs, IGF-binding proteins and IGFBP-proteases. Acta Physiol Hung 92: 97-107, 2005.

40. van Bunderen CC, Oosterwerff MM, van Schoor NM, Deeg DJ, Lips P and Drent ML: Serum IGF1, metabolic syndrome, and incident cardiovascular disease in older people: A population-based study. Eur J Endocrinol 168: 393-401, 2013.

41. van Nieuwpoort IC, Vlot MC, Schaap LA, Lips P and Drent ML: The relationship between serum IGF-1, handgrip strength, physical performance and falls in elderly men and women. Eur $\mathbf{J}$ Endocrinol 179: 73-84, 2018

42. Lam CS, Chen MH, Lacey SM, Yang Q, Sullivan LM, Xanthakis V, Safa R, Smith HM, Peng X, Sawyer DB, et al: Circulating insulin-like growth factor-1 and its binding protein-3: Metabolic and genetic correlates in the community. Arterioscler Thromb Vasc Biol 30: 1479-1484, 2010.

43. Saydah S, Ballard-Barbash R and Potischman N: Association of metabolic syndrome with insulin-like growth factors among adults in the US. Cancer Causes Control 20: 1309-1316, 2009. 